\title{
Physics Impact of the SDC Endcap Hadronic Cracks
}

\author{
Dan Green \\ Fermi National Accelerator Laboratory \\ P.O. Box 500, Batavia, Illinois 60510
}

May 1993 


\section{Disclaimer}

This report was prepared as an account of work sponsored by an agency of the United States Government. Neither the United States Government nor any agency thereof, nor any of their employees, makes any warranty, express or implied, or assumes any legal liability or responsibility for the accuracy, completeness, or usefulness of any information, apparatus, product, or process disclosed, or represents that its use would not infringe privately owned rights. Reference herein to any specific commercial product, process, or service by trade name, trademark, manufacturer, or otherwise, does not necessarily constitute or imply its endorsement, recommendation, or favoring by the United States Government or any agency thereof. The views and opinions of authors expressed herein do not necessarily state or reflect those of the United States Government or any agency thereof. 


\title{
PHYSICS IMPACT OF THE SDC ENDCAP HADRONIC CRACKS
}

\author{
Dan Green \\ Fermi National Accelerator Laboratory \\ Batavia, IL.
}

April 1993

\section{Introduction}

The SDC calorimeter has a set of design requirements [1]. Among them is the need for "hermeticity". The calorimeter should not register particle energies which are so badly mismeasured as to induce a significant missing energy. Such a mismeasure would mimic the existence of a neutrino in the event. The extreme case of a catastrophic mismeasure of particle energy is the total failure to register the energy of a particle due to its loss in a crack or "dead" region of the calorimeter.

The baseline design of the SDC endcap calorimeter consists of a crackless, monolithic electromagnetic (EM) compartment, followed by an azimuthal array of 1/16 "wedge" hadronic (HAD) modules. Of necessity, tolerances in construction lead to azimuthal cracks between adjacent wedge modules. The purpose of this note is to examine the Physics impact of these cracks, and to examine, in detail, their allowable extent.

\section{Location in Depth of the Dogleg}

Clearly, since a hadronic shower develops both transversely and longitudinally, a reasonable model of such development must be made. The longitudinal development of a hadronic shower is modeled using the UA1 parametrization [2]. For an electron or photon shower, the Particle Data Group representation was used [3]. Details of the assumptions can be found in a previous study of the impact of the SDC barrel crack on the Physics [4].

For both electromagnetic and hadronic showers, one of the major fluctuations in the shower development is the stochastic nature of the interaction point. In addition, the hadronic showers vary from shower to shower as to their initial neutral pion content. For 
this study, the UA1 fit was fluctuated in neutral content by $\langle$ fo $>=0.46$, dfo $=0.15$ on an event by event basis.

The initial design choice for the crack is at what depth to put the "dogleg". The idea is to minimize the dead material intercepted by the shower by making a transverse "dogleg" in the crack at the midpoint of the shower development in depth. A hadron of $5 \mathrm{TeV}\left(\mathrm{P}_{\mathrm{t}}=\right.$ $0.5 \mathrm{TeV}$ at $|\eta|=3$, where the effect of azimuthal cracks is most important) was chosen. In Fig. 1 is shown the integral depth distribution of the energy as a function of the depth in $\mathrm{cm}$ of Fe with respect to the conversion point. The scatter of points comes from the evaluation of the integral with variable values of the fo parameter, as discussed above. This procedure gives some qualitative idea of the effect of fo fluctuations.

Clearly, placing the dogleg at a depth of $\sim 3$ interaction lengths with respect to the conversion point, would roughly equally split the energy in depth between the front and rear parts of the dogleg. In what follows, the depth of the dogleg is fixed at 3 interaction lengths in HAD. The depth of the front EM compartment is $\sim 1$ interaction length, so that it is expected that the hadronic shower will split its energy equally in the front and rear parts of the dogleg. The average values of the energy fraction in the various compartments is $<\mathrm{EM}>=1.5 \%,<\mathrm{H} 1>=49 \%,<\mathrm{H} 2>=45 \%$. At a depth of 10 interaction lengths, there is a $4.5 \%$ leakage energy on the average.

\section{Transverse Distribution of Hadronic Showers}

The other 2 basic design choices are the transverse width of the crack, and the transverse "throw" of the dogleg. The design of the endcap hadronic wedge (ECHAD) leads to an estimated scintillator to scintillator steel width of $1 \mathrm{~cm}$ [5]. In most of what follows, a $2 \mathrm{~cm}$ dead width is assumed, leading to a factor of 2 pessimism/realism factor over the design value.

Firstly, the transverse distribution of the shower energy as a function of depth is parameterized. Some representative data is shown in Fig. 2. Clearly, the full width of a shower increases roughly linearly as the depth with respect to the conversion point [6]. For the data shown in Fig. 2, the FWHM in $\mathrm{cm}$ of Fe is $\sim 2.5$ times the depth in interaction lengths. The transverse distribution cannot be represented by a single Gaussian or exponential [7]. The parametrization chosen was to represent the distribution as a sum of 2 exponentials, characterized by a mean length of $2.2 \mathrm{~cm}$ and $7.0 \mathrm{~cm}$ respectively. Further, it is assumed that the 2 mean lengths both scale linearly in depth. 
The Monte Carlo distribution of the transverse (x) distribution in $\mathrm{cm}$ of $\mathrm{Fe}$ is shown in Fig. 3, at a depth of 3 interaction lengths. The 2 component behavior is quite evident. It has been assumed that the $\mathrm{x}$ distribution is energy independent. The justification for this simplifying assumption is not strong. The FWHM seen in Fig. 3 is $\sim 7 \mathrm{~cm}$, in agreement with Fig. 2.

Single hadrons of $5 \mathrm{TeV}$ energy were thrown at the calorimeter incident on the center line of an inert crack of variable width. It is assumed that energy deposited in the crack is lost. The fraction found outside the crack at a depth of 3 interaction lengths in Fe, as a function of the crack width, is shown in Fig. 4. Fig. 4 is the integral of Fig. 3. Clearly, for a FWHM crack of $2 \mathrm{~cm}$ (twice the design goal), 15\% of the energy is lost. Note that this is the maximum (on axis) fraction of energy that can be lost. It requires a crack of FWHM equal to $8 \mathrm{~cm}$ before half of the energy is lost. Using Fig. 4 , one can scale the subsequent results should the crack not be $2 \mathrm{~cm}$ wide.

\section{Single Hadron Response}

Single hadrons were then used to probe the transverse response of the SDC toy model calorimeter. Hadrons of $5 \mathrm{TeV}$ were incident on a monolithic EM compartment, followed by a 9 interaction length HAD compartment. The mean energy response is shown in Fig. 5a as a function of the HAD crack width for incidence on the HAD crack centerline. The crack has no dogleg in this study. Clearly, hadrons leak 5\% at this energy.

The rms of the energy distribution as seen in the calorimeter is shown in Fig. $5 \mathrm{~b}$. Clearly, leakage fluctuations lead to an rms of $1.7 \%$ in the absence of any inert crack. The $5 \%$ leakage factor is already evident in Fig. 1. In agreement with Fig. 4, the data in Fig. 5a indicate a $10 \%-15 \%$ loss for a $2 \mathrm{~cm}$ FWHM crack in the HAD compartment. The loss additional to the leakage is roughly linear in the crack width. The slope is $\sim 7 \%$ lost $/ \mathrm{cm}$ FWHM of crack.

As seen in Fig. 5b, a crack FWHM of $>3 \mathrm{~cm}$ leads to a rms energy measurement error which is $>4 \%$. These additional induced errors are outside the SDC requirements [1]. The origin of these fluctuations in the energy measurement is the fluctuations in transverse shower development which are built into the Monte Carlo model.

Single hadrons are used to evaluate the "throw" of the dogleg. The dogleg is shown in Fig. 6 for a $6 \mathrm{~cm}$ "throw". The comparison is made to a crack without any "throw". The dogleg depth is chosen to be at a depth of 3 interaction lengths, as justified using the data given in Fig. 1. The dogleg width is now fixed at $2 \mathrm{~cm}$, twice the design 
goal. The "throw" is set to be $\sim$ the FWHM of the hadronic shower at that depth. In this way, one attempts to reduce the energy loss of any given shower. Clearly, this reduction is purchased at the cost of energy loss over a larger range of transverse impact parameters.

The mean calorimeter energy divided by the incident energy as a function of the incident particle position with respect to the HAD1 crack (see Fig. 6) is shown in Fig. 7. For the case of no dogleg, the response dips to an incremental 15\% loss (recall the 5\% leakage loss). The biggest dip of $15 \%$ agrees with the plot of Fig. 5a. The FWHM of the crack response extends over a FWHM of $7 \mathrm{~cm}$ (in agreement with Fig. 3). For the doglegged crack shown in Fig. 6, the response is made shallower and wider. The incremental maximum dip is now $10 \%$, extending over a FWHM of $10 \mathrm{~cm}$.

There is a second argument for a dogleg construction. Tolerance errors in construction means that there will not be solid steel in the crack. Thus, in order to insure shower initiation, dogleg construction is required. The "throw" of the dogleg is clearly not a crisply defined optimization problem. The design given here appears to be adequate.

\section{Jet Response}

Jets of $5 \mathrm{TeV}$ were constructed and thrown at the calorimeter. Details of the jet building are given in Ref. 4. The jets are the physical objects of interest. However, they are more diffusely spatially defined than the single hadrons which have been used to probe the crack so far. Thus, lessened sensitivy to the crack is expected.

In contrast to single hadrons, the jet deposits a larger fraction of its energy in the EM compartment; $\langle\mathrm{EM}>=33 \%,\langle\mathrm{H} 1>=43 \%,<\mathrm{H} 2\rangle=24 \%$. The jet leakage is only $1.5 \%$, in contrast to the $5 \%$ single hadron leakage.

Jets far from the dogleg have $98 \%$ of the $5 \mathrm{TeV}$ energy captured on average. The fractional jet energy has an rms of $2.3 \%$ due to leakage and crack absorption. In contrast, a $5 \mathrm{TeV}$ jet incident on the centerline of the dogleg shown in Fig. 6 has a mean of $92 \%$ of its energy detected, with an rms of $2.6 \%$. Therefore, the jet suffers an incremental loss of, at most, $6 \%$ if its axis is coincident with the dogleg centerline. Surely, that level of effect can be easily corrected for by mapping. 


\section{Summary}

The effect of a "doglegged" crack on the response of the SDC calorimeter to incident jets (the physically relevant quantities) has been examined. For a crack of design width $(1 \mathrm{~cm})$, the maximum incremental loss in fractional mean energy is $\sim 3 \%$. The maximum incremental induced rms energy error is $<1 \%$. These induced nonlinearities and errors are well within the SDC design requirements [1] and are partially correctable.

\section{References}

1. SDC Technical Design Report, SDC-92-101 (1992).

2. R.K. Bock et al., CERN-EP180-206 (1980).

3. Review of Particle Properties, Phys. Rev. D 45 (1992).

4. D. Green, "The Effects of Cracks on Calorimeter Response in the SDC "Dogleg" Design', Fermilab-TM-1810 (1992).

5. Igor Churin, private communication

6. S. Iwata, DPNU-3-79, Feb. 1979.

7. V.A. Davidov et al., Nuc. Inst. Meth. 174369 (1980). 


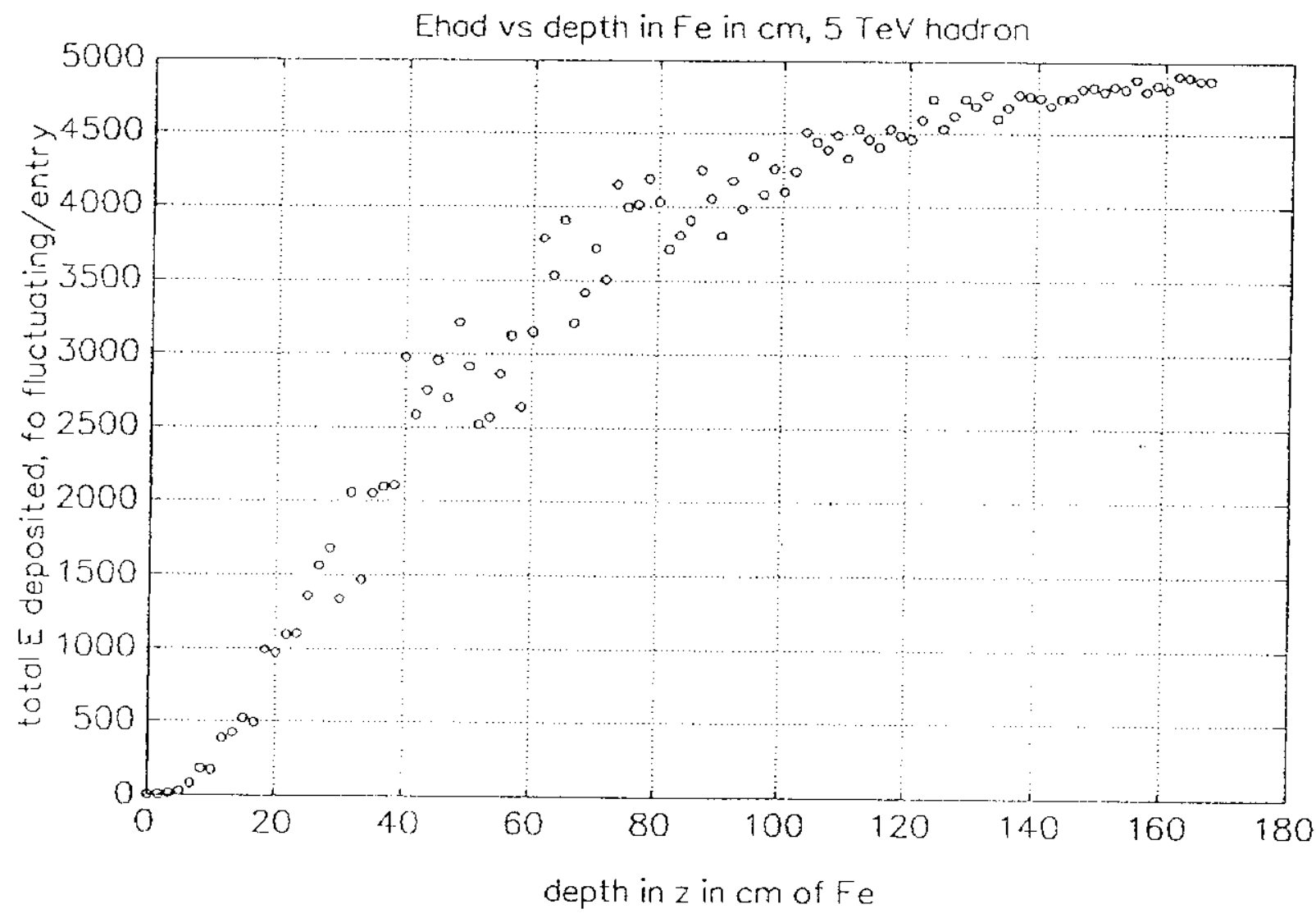

Fig. 1. Total deposited energy up to depth $\mathrm{z}$, in $\mathrm{cm}$ of Fe, with respect to the interaction point for an incident hadron of $5 \mathrm{TeV}$ energy. The point to point fluctuations are due to the fluctuations in the neutral fraction chosen for each point, fo $=0.46 \pm$ 0.15 . 


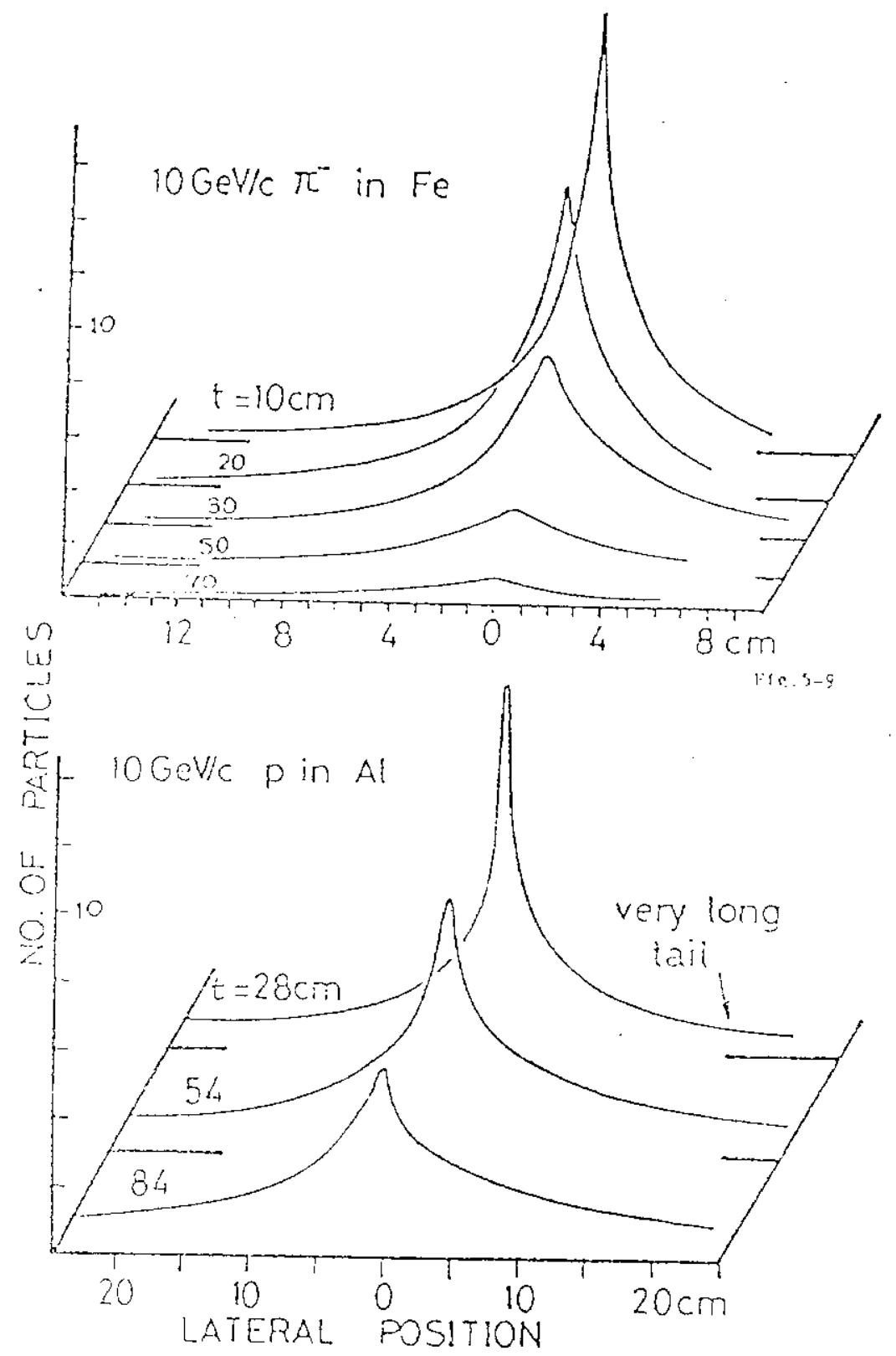

Fig. 2. Distribution of tranverse positions for $10 \mathrm{GeV}$ hadrons incident on $\mathrm{Fe}$ and $\mathrm{Al}$ detectors. The transverse evolution of the showers with depth is illustrated. The FWHM of the shower is roughly proportional to the depth of the shower. 


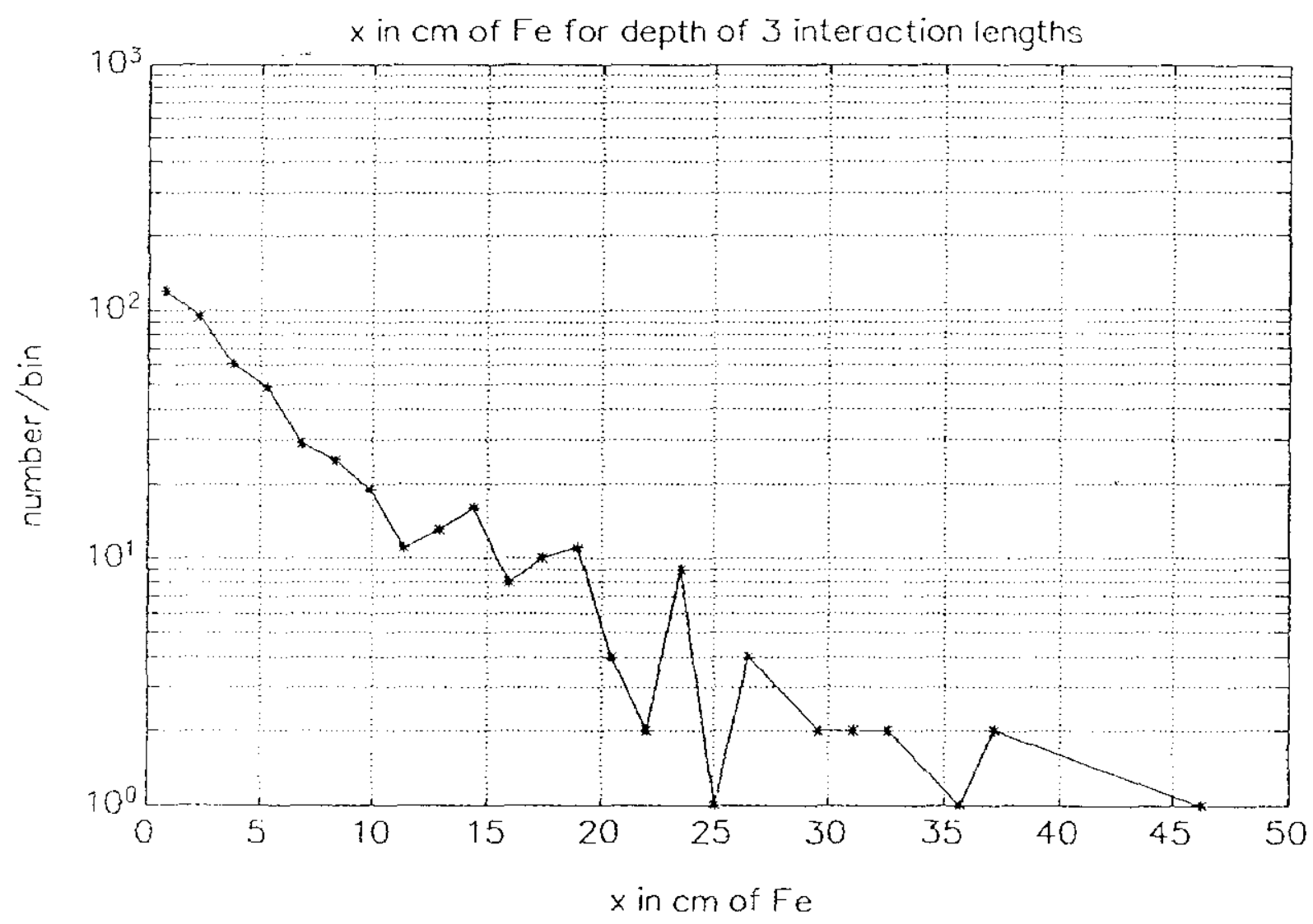

Fig. 3. Monte Carlo distribution of the transverse position of a $5 \mathrm{TeV}$ hadron at a depth of 3 interaction lengths. The 2 characteristic exponential lengths are evident. 


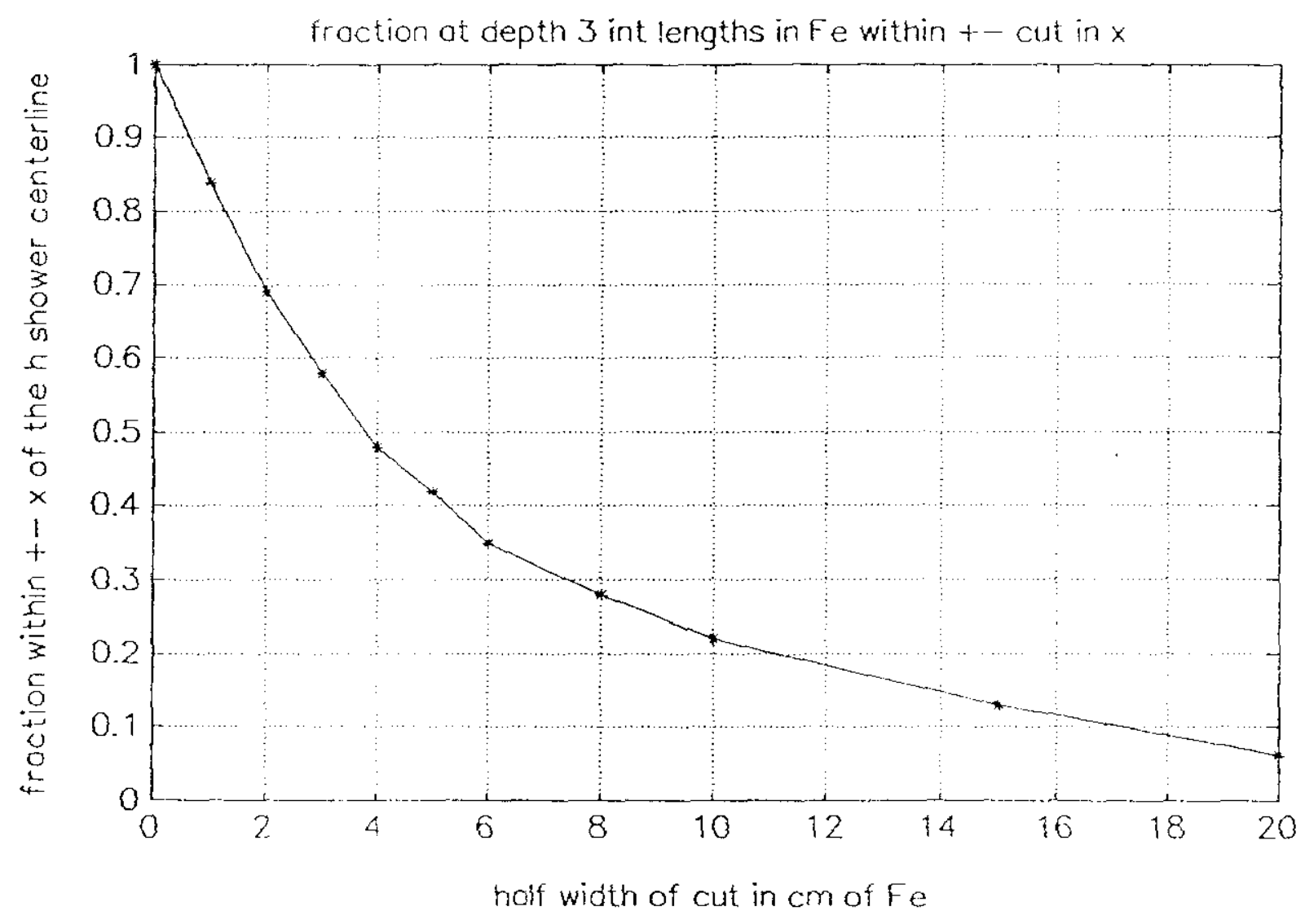

Fig. 4. Monte Carlo distribution of the fraction of energy in a hadronic shower contained outside a cut, in $\mathrm{cm}$ of $\mathrm{Fe}$, at a depth of 3 interaction lengths. 

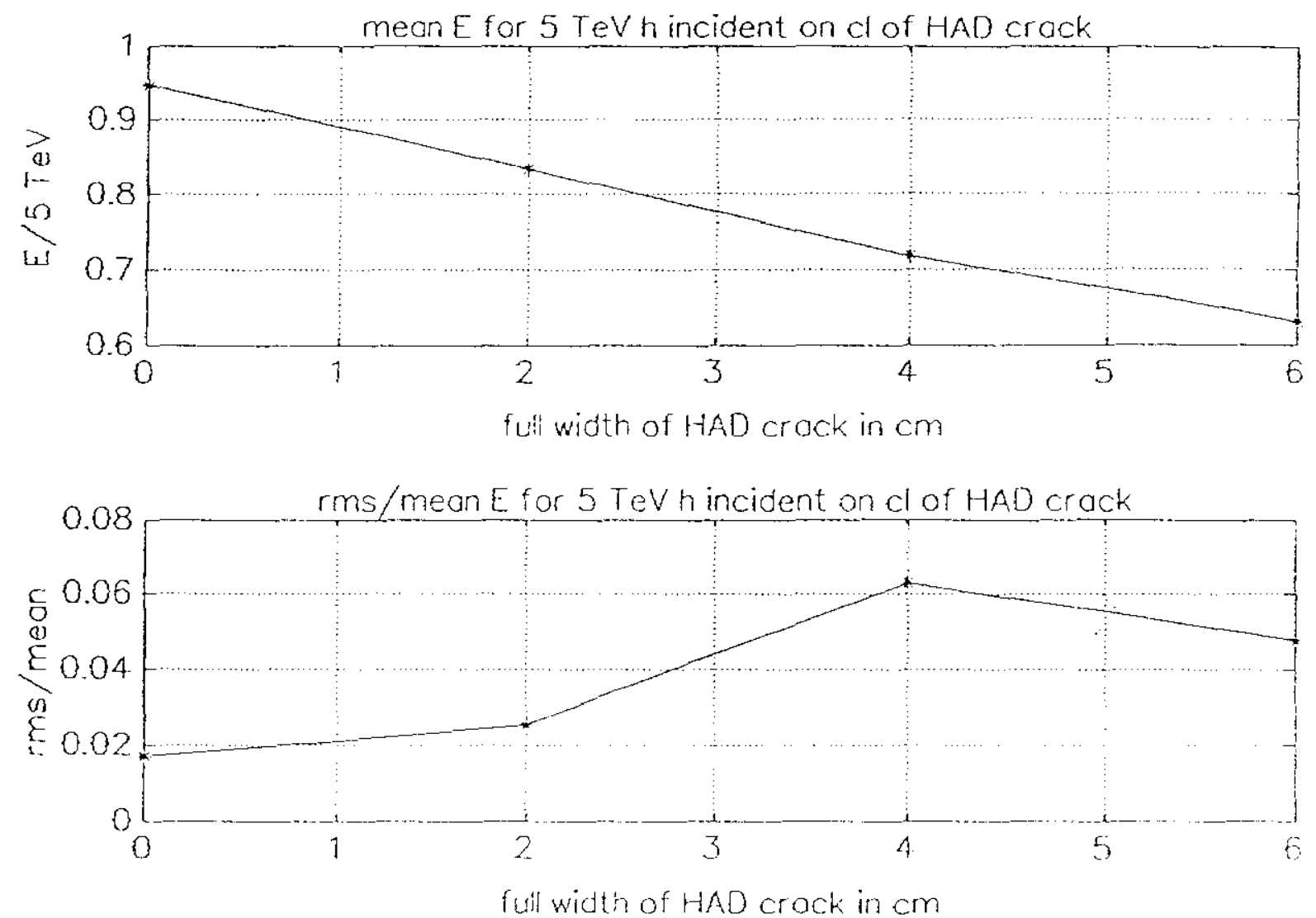

Fig. 5. Monte Carlo results for a $5 \mathrm{TeV}$ hadron incident on a crack as a function of the FWHM of that crack in $\mathrm{cm}$ of Fe. The hadron is incident on the center line of the crack.

a. Mean fractional energy deposited outside the crack.

b. rms / mean of the observed energy deposited outside the crack. 


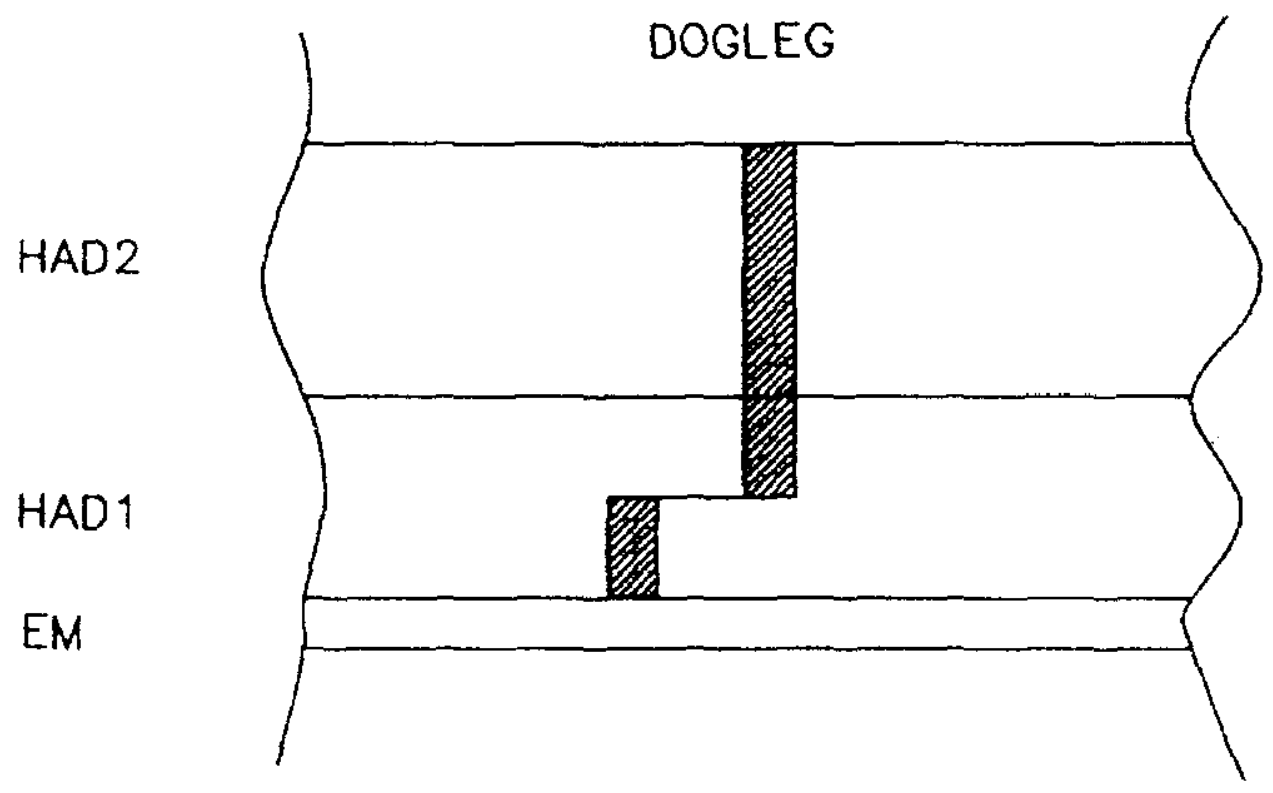

Fig. 6. Schematic of the Monte Carlo model of the doglegged crack. The model is defined by the width of the crack, the depth location of the dogleg, and the transverse "throw" of the dogleg. 


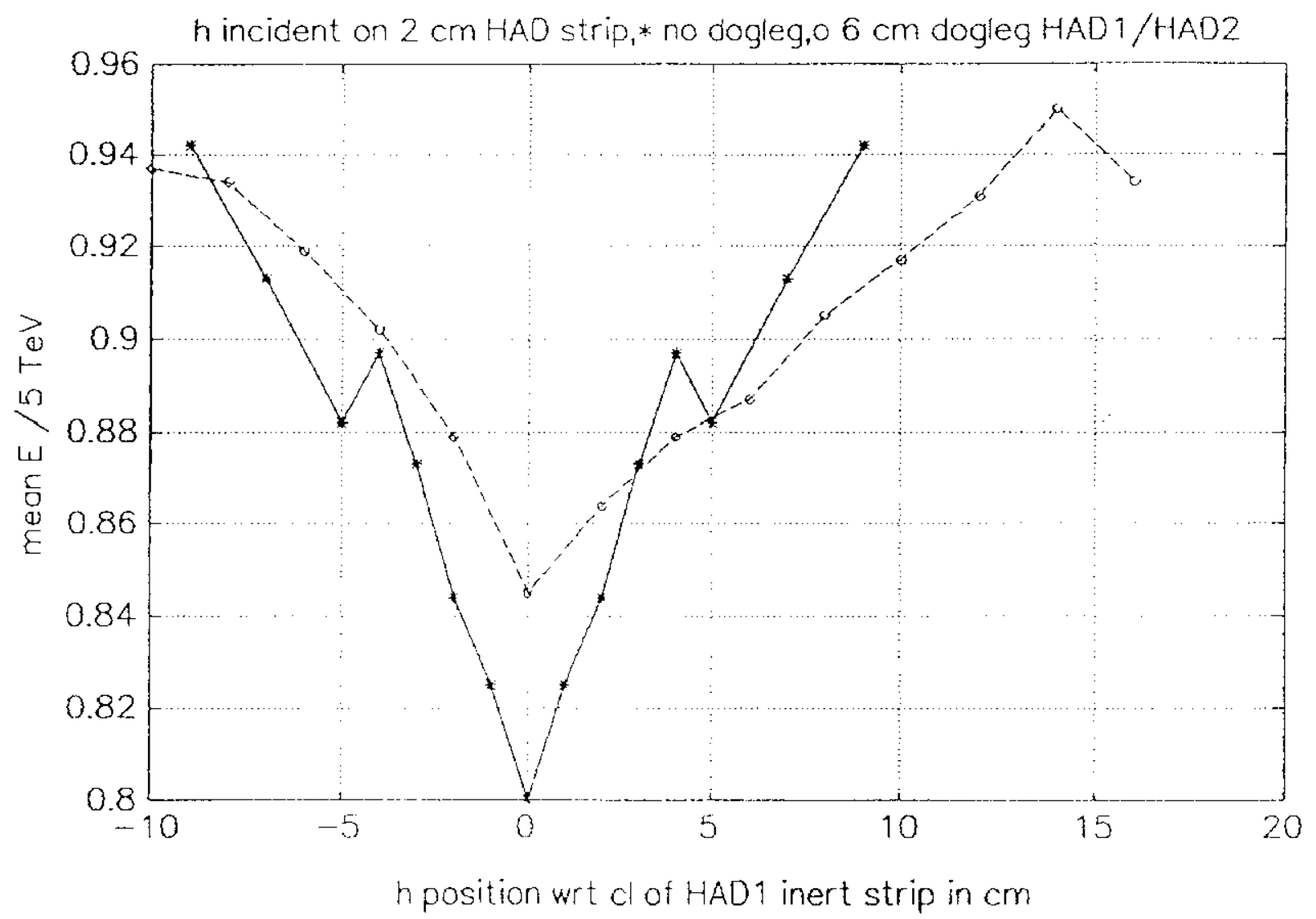

Fig. 7. Response of a $5 \mathrm{TeV}$ hadron as a function of the position of incidence with respect to the center line of the upstream portion of the crack. The crack width is $2 \mathrm{~cm}$, and the transverse dogleg occurs at a depth of 3 interaction lengths in the steel, following the EM (monolithic and hermetic) compartment. The 2 curves refer to * - no transverse "throw" and o- a $6 \mathrm{~cm}$ transverse "throw" of the dogleg. 